\title{
BMJ Open Protocol for a double-blind placebo- controlled trial to evaluate the efficacy of probiotics in reducing antibiotics for infection in care home residents: the Probiotics to Reduce Infections iN CarE home reSidentS (PRINCESS) trial
}

Eleri Owen-Jones, ${ }^{1}$ Rachel Lowe, ${ }^{1}$ Mark Lown, ${ }^{2}$ David Gillespie, ${ }^{1}$ Katy Addison, ${ }^{1}$ Tony Bayer, ${ }^{3}$ Philip C Calder, ${ }^{4,5}$ Jane Davies, ${ }^{1}$ Mina Davoudianfar, ${ }^{6}$ James Downs, ${ }^{7}$ Alison Edwards, ${ }^{1}$ Nick A Francis, ${ }^{3}$ Richard Fuller, ${ }^{2}$ Richard Hobbs, ${ }^{6}$ Kerenza Hood, ${ }^{1}$ Mandy Lau, ${ }^{1}$ Paul Little, ${ }^{2}$ Michael Moore, ${ }^{2}$ Victoria Shepherd, ${ }^{3}$ Helen Stanton, ${ }^{1}$ Alun Toghill, ${ }^{7}$ Mandy Wootton, ${ }^{8}$ Chris C Butler ${ }^{6}$

To cite: Owen-Jones E, Lowe R, Lown M, et al. Protocol for a double-blind placebocontrolled trial to evaluate the efficacy of probiotics in reducing antibiotics for infection in care home residents: the Probiotics to Reduce Infections iN CarE home reSidentS (PRINCESS) trial. BMJ Open 2019;9:e027513. doi:10.1136/ bmjopen-2018-027513

- Prepublication history for this paper is available online. To view these files, please visit the journal online (http://dx.doi org/10.1136/bmjopen-2018027513)

Received 29 0ctober 2018 Revised 21 February 2019 Accepted 22 February 2019

Check for updates

(c) Author(s) (or their employer(s)) 2019. Re-use permitted under CC BY-NC. No commercial re-use. See rights and permissions. Published by BMJ.

For numbered affiliations see end of article.

Correspondence to

Dr Eleri Owen-Jones;

Owen-JonesCE@cardiff.ac.uk

\section{ABSTRACT}

Introduction Care home residents are at increased risk of infections and antibiotic prescription. Reduced antibiotic use from fewer infections would improve quality of life. The Probiotics to Reduce Infections iN CarE home reSidentS (PRINCESS) trial aims to determine the efficacy and investigate mechanisms of daily probiotics on antibiotic use and incidence of infections in care home residents. Methods and analysis PRINCESS is a double-blind, individually randomised, placebo-controlled trial that will assess the effect of a daily oral probiotic combination of Lactobacillus rhamnosus, GG (LGG) and Bifidobacterium animalis subsp. lactis, BB-12 (BB-12) on cumulative antibiotic administration days (CAADs) (primary outcome) for infection in up to 330 care home residents aged $\geq 65$ years over up to 12 months. Secondary outcomes include: Infection: Total number of days of antibiotic administration for each infection type (respiratory tract infection, urinary tract infection, gastrointestinal infection, unexplained fever and other); number, site, duration of infection; estimation of incidence and duration of diarrhoea and antibioticassociated diarrhoea; Stool microbiology: Clostridium difficile infection; Gram-negative Enterobacteriaceae and vancomycin-resistant enterococci; LGG and BB-12. Oral microbiology: Candida spp. Health and well-being: Self and/or proxy health-related quality of life EQ5D (5L); selfand/or proxy-reported ICEpop CAPability measure for older people. Hospitalisations: number and duration of all-cause hospital stays. Mortality: deaths. Mechanistic immunology outcomes: influenza vaccine efficacy (haemagglutination inhibition assay and antibody titres); full blood count and immune cell phenotypes, plasma cytokines and chemokines; cytokine and chemokine response in whole blood stimulated ex vivo by toll-like receptor 2 and 4 agonists; monocyte and neutrophil phagocytosis of Escherichia coli; serum vitamin D.

Ethics and dissemination Ethics approval is from the Wales Research Ethics Committee 3. Findings will

\section{Strengths and limitations of this study}

Relevant information on an under-researched population in a growing sector of care.

- Option for participants to opt out of aspects of sample collections, potentially enhancing recruitment and thus generalisability.

- Data will be collected prospectively on a weekly basis for each trial participant by blinded research nurses visiting care homes.

- Recruiting to our target will be a challenge especially as we will approach consultees for participants who lack capacity to consent for themselves.

- Recent antimicrobial stewardship guidance specifically for long-term care facilities could result in lower antibiotic prescribing rates during the trial.

be disseminated through peer-reviewed journals and conferences; results will be of interest to patient and policy stakeholders.

Trial registration number ISRCTN16392920; Pre-results.

\section{INTRODUCTION}

Care home residents are at increased risk of infections due to weakened immunity, close-proximity living and multimorbidity and are prescribed antibiotics for an average of almost 20 days each year, far more than the general population. ${ }^{12}$ High antibiotic use increases the risk of colonisation and infection with antimicrobial-resistant (AMR) organisms that may then cycle within care homes and spread between hospitals and community. Infections in care home residents cost the 
NHS $>£ 54$ million/year in hospitalisation alone ${ }^{3}$ and are the most common reason for residents to be hospitalised. AMR Gram-negative septicaemia and AMR urinary tract infection (UTI) are on the increase in the community, especially among older people. ${ }^{45}$ Recent consumption of antibiotics is the greatest risk factor for carriage and infection with AMR bacteria even after controlling for age, comorbidity and other risk factors. ${ }^{67}$ AMR infections are generally more serious and are associated with longer hospital stays, increased costs and increased risk of poor outcomes including death, particularly in older people. ${ }^{8}$ Evidence-based interventions are needed to improve the quality of life through reducing the incidence of common infections and antibiotic use. ${ }^{9}$ Other than vaccination and hygiene methods, there are few interventions proven to prevent infection in care homes, and even so-called 'minor' infections can have an important negative impact on health, well-being and dignity, especially for older, frail people.

Probiotics are defined by the WHO as 'live microorganisms which when administered in adequate amounts confer a health benefit to the host'. ${ }^{10}$ They may prevent infection by blocking pathogenic colonisation and enhancing gut-immune interaction, with influence on mucosal and systemic immunity, leading to enhanced natural killer cell activity and vaccine response ${ }^{11}$ in older people. ${ }^{12}{ }^{13}$ A systematic review and meta-analysis found that probiotics reduced the risk of upper respiratory tract infections (URTIs) and reduced antibiotic prescribing in adults and that side effects were minor. ${ }^{14}$ However, the review noted poor allocation concealment in several studies and heterogeneity in findings and recommended that future randomised controlled trials (RCTs) should 'focus on older people'. Meta-analysis indicates that probiotics can reduce antibiotic-associated diarrhoea (AAD), but more research is needed to determine which probiotics are associated with greatest efficacy and for which patients receiving which specific antibiotics. ${ }^{15}$ Probiotics may also reduce AMR colonisation, ${ }^{14}$ which could be important as most UTIs arise from autoinoculation with gut organisms.

Effects of probiotics are thought to vary by strain due to differing resistance to gastric acid and bile, ability to colonise mucosa and susceptibility to antibiotics. Lactobacillus rhamnosus, GG (LGG) is the most studied probiotic, including in elderly populations, and has been found to be safe. In a meta-analysis of RCTs involving 1805 children, LGG reduced the risk of otitis media, URTI and antibiotic use. ${ }^{16}$ LGG may act as an immune adjuvant to influenza vaccination ${ }^{17}$ and benefit oral health. ${ }^{18}$ Many studies have utilised several strains of probiotic simultaneously (which may increase efficacy due to differing modes of action), and LGG and Bifidobacterium animalis subsp. lactis, BB-12 (BB-12) have been included in the same probiotic formulation. ${ }^{19} 20$ The combination probiotic reduced severity and duration of URTIs in 231 college students. ${ }^{21}$ The combination probiotic, and BB-12 alone, have been shown to reduce URTIs and otitis media in infants $^{22} 23$ and to reduce symptoms of irritable bowel syndrome and stabilise bowel microbiota in adults. ${ }^{24}$ However, neither probiotic strain has been evaluated primarily in care home residents. Although probiotics are effective in preventing $\mathrm{AAD},{ }^{15}{ }^{25}$ evidence is lacking on the prevention of all-cause common infections and antibiotic prescribing in care homes.

\section{METHODS AND ANALYSIS \\ Objectives}

PRINCESS aims to evaluate effectiveness of daily administration of a combination of two probiotics (LGG and BB-12) on total days on antibiotics for infections, incidence and severity of infections, and on a range of secondary and related mechanistic outcomes.

\section{Design and setting}

A double-blind, individually randomised placebo-controlled trial. An internal pilot will assess care home recruitment, data collection, study product administration and sampling procedures. Participants are care home residents in approximately 20 care homes in Wales and England.

\section{Participants}

Inclusion criteria

- Currently living in a care home setting (residential, nursing or mixed).

- Willing and able to give informed consent for participation in the trial OR if the participant lacks capacity, a consultee willing to complete a consultee declaration form for participation on their behalf.

- Aged 65 years or older.

\section{Exclusion criteria}

- Known to be immunocompromised (requiring immune-suppressants, long-term, high-dose, oral, intramuscular or intravenous steroids).

- Currently taking regular probiotics and is not willing to adapt to trial protocol.

- Currently participating in a clinical trial of an investigational medicinal product (CTIMP) or has been a participant in a CTIMP in the last 30 days.

- A temporary care home resident (ie, less than 1 month of planned transitional/respite residential care).

- Death is thought to be imminent.

- Is lactose intolerant.

\section{Informed consent}

Informed consent or a consultee declaration (see below) will be obtained prior to any trial procedures. Where there are concerns that a resident may have impaired mental capacity, a mental capacity assessment will be undertaken by a qualified research nurse (RN) in accordance with the Mental Capacity Act 2005. Eligible residents may participate in the trial even if they (or their consultee) prefer to opt out from providing blood and/or stool and/or saliva samples. Residents who provide informed consent 
at trial entry will be asked to agree to remain in the study in the event of a loss of capacity. The PRINCESS RNs will be trained in how to assess capacity, and trial participants will be (re)assessed regularly. Trial participant's general practitioner (GP) will be informed of their participation in the PRINCESS trial.

\section{Residents lacking capacity}

Where a resident lacks capacity to provide consent for themselves, a family member or friend will be consulted and will act as their 'consultee'. If a consultee who has an unpaid or non-professional role in care cannot be identified, or is not willing to act, a nominated consultee will be appointed and consulted prior to including the resident in the trial. If a consultee themselves loses capacity or dies during the trial period, an alternative family member or friend will be contacted to act as consultee and a new consultee declaration form will be completed. If there is no one to represent the resident concerning the trial, they will be ineligible or withdrawn if already recruited. If agreement for the resident to participate is provided by a consultee due to lack of capacity and they regain capacity during the trial, the participant will be fully informed and consent to remain in the trial will be sought from the participant themselves. If a consultee is required and they cannot attend a face-to-face interview, the above documents may be sent from and returned to the care homes by post.

\section{Verbal consent/declaration}

If a resident or a consultee cannot read or provide handwritten signatures on the consent/declaration form, verbal consent/agreement will be taken. In such cases, a delegated individual will read the trial information sheet to them and discuss the trial to ensure understanding. A member of the research team will witness, sign and date the consent/declaration form to confirm that valid consent or a consultee declaration has been given.

\section{Withdrawal}

Participants may withdraw/be withdrawn from the trial at any time for any reason without prejudice to future care, and with no obligation to give a reason, and will be given an option as to the level of withdrawal of data.

\section{Randomisation}

Participants will be remotely randomised using an online system developed and hosted by the University of Oxford Primary Care Clinical Trials Unit (Sortition). Participants will be randomised to either probiotic or placebo in a 1:1 ratio using minimisation. Allocations will be balanced by care home and resident gender. A random component, set at $80 \%$, will maintain integrity of the allocation process. As PRINCESS is a double-blind trial, the participants, care home staff, treating clinicians and trial team (including the trial statistician and RNs conducting all assessments) will be unaware of the group to which the participant has been allocated for the duration of the trial. If unblinding is needed, the procedure will be documented and performed by the independent statistician, and as a back-up the Centre for Trials Research (CTR) pharmacovigilance and safety team will also be trained to perform this function.

\section{Intervention}

Participants will be asked to take an oral dose of probiotic (LGG and BB-12) or a matched placebo (containing maltodextrin, microcrystalline cellulose, magnesium stearate and silicon dioxide) as a capsule once daily for up to 12 months. The manufacturer of study products is Chr. Hansen A/S, Bøge Alle 10-12, 2970 Hørsholm, Denmark. The dates of manufacture of study products are December 2015 (total cell count per capsule $1.6 \times 10^{10}$ ) and March 2017 (total cell count per capsule $1.3 \times 10^{10}$ ).

The probiotic or placebo (referred to as study product) will be administered by the resident's normal caregiver. The preferred route of administration will be as follows:

1. The capsule swallowed whole with water.

2. Capsule will be emptied into a small amount of cold or lukewarm liquid and swallowed.

3. Capsule contents will be sprinkled onto cold or lukewarm (not hot) food and eaten.

The capsules will be suitable for vegans, are Halal and Kosher (Kosher dairy excluding Passover), will not contain any genetically modified organisms or raw materials and allergen labelling will not be required. The capsules will contain lactose. They will be stable at room temperature for 2 years and temperature monitoring will not be required for short-term (eg, 1 month) storage. Participants admitted to hospital will not be expected to continue taking study product during their hospital stay. Data regarding participants' adherence to study product will be collected from several sources including medication administration record sheets and regular capsule counts (of unused study product). Stool culture will also be used to determine the presence of probiotic organisms in the bowel of participants who provide consent for these samples at baseline, 3 and up to 12 months. This will give an indication of adherence, survival of the probiotic in the large bowel and potential contamination in the placebo arm. Study product will be continued even when on antibiotics and other medications.

\section{Outcomes}

\section{Primary outcome}

Cumulative systemic antibiotic administration days (CAAD) for all-cause, acute infections; total number of days of systemic antibiotic administration as recorded in care home medical records and discharge summaries if the participant is admitted to hospital, collected by the RNs.

\section{Secondary outcomes}

Infection

- Total number of days of antibiotic administration for each infection type as recorded in care home medical records (collected by RNs). 
- Number, site, duration (mean and cumulative) of infection, as recorded in care home medical records (collected by RNs).

- Estimation of incidence and duration of diarrhoea when taking (and not taking) oral antibiotic treatment and AAD (question asked by RNs).*

- Stool sample laboratory analysis

- Prevalence of Clostridium difficile**infection.

- Culture and antibiotic sensitivity of Gram-negative Enterobacteriaceae and vancomycin-resistant enterococci.

- Levels of LGG and BB-12.

\section{Oral microbiology}

- Semiquantitative analysis of oral rinse or saliva samples for Candida spp.

\section{Health and well-being}

- Self and/or proxy-reported health-related quality of life measurement EQ-5D (5L).

- Self-and/or proxy-reported ICEpop CAPability measure for Older people (ICECAP-O).

\section{Hospitalisations}

- Number and duration of hospital stays for all-cause hospitalisation (as recorded in care home records and discharge summaries, collected by RNs).

\section{Mortality}

- Deaths (from care home records, collected by RNs).

\section{Mechanistic immunology outcomes}

- Influenza vaccine efficacy (haemagglutination inhibition assay and antibody titres).

- Full blood count.

- Immune cell phenotypes, plasma cytokine and chemokine concentrations, cytokine and chemokine responses in whole blood samples stimulated ex vivo by toll-like receptor 2 and 4 agonists and leucocyte phagocytosis of Escherichia coli.

\section{Tertiary outcomes}

Level of serum vitamin D and AMR colonisation within stool sample.

*Diarrhoea is defined as: 'the abnormal passing of loose or liquid stools, with increased frequency and/or increased volume' (National Institute for Health and Care Excellence (NICE) Clinical Knowledge Summaries). However, the norm for the participant will also considered when collecting this information as care home residents often have loose stools as a result of overflow or aperient use rather than an infective cause. Stool charts are kept in care homes that usually record stool consistency based on the Bristol Stool Chart, and our RNs will have access to these charts. We have trained our RNs to record the presence of loose stools. AAD will be defined as diarrhoea occurring following administration of antibiotics and up to 8 weeks after stopping antibiotic treatment. $^{2}$
**We will look at the presence of $C$. difficile in the stool as a risk factor for further disease, which could be influenced by probiotics.

\section{Sample size}

Primary outcome

The original target for the PRINCESS trial was to randomise 330 participants from around 20 care homes in Wales and England for $90 \%$ power at the $5 \%$ level to demonstrate a $10 \%$ relative reduction in CAADs (assuming an average CAAD of 17.4 days and an absolute reduction in the probiotic arm to 15.6 days per resident-year). We consider that a $10 \%$ reduction is feasible and would be clinically important.

This sample size accounted for $30 \%$ of participants being lost to follow-up due to withdrawal or death during the study.

An interim assessment (to determine if we met the Stop/Go contractual criteria) of primary outcome ascertainment revealed that the mean percentage of days for which there are valid antibiotic administration data (ie, either no antibiotics administered or the number of days on which antibiotics have been administered in each week) is $77.4 \%$ or 283 days out of a possible 365 on average. This percentage varies depending on the length of time participants have been in the study. However, it remains high for participants in the study for over 6 months and does not consider other data sources that might be used (eg, hospital discharge summaries, medication administration records). This is likely therefore to underestimate availability of primary outcome data, but nevertheless is a more accurate reflection of the likely level of follow-up when compared with our original assumption.

Given slower than anticipated recruitment, and this new information regarding the trade-off between numbers of participants required and average length of follow-up, we will aim to randomise between 258 and 270 participants. Assuming a mean number of days for which primary outcome data will be available (ie, accounting for follow-up time and missing data) of approximately 250 days, this will provide at least $82 \%$ power to detect a $10 \%$ relative reduction in CAAD.

\section{Secondary mechanistic outcomes}

Previous research has found a $40 \%$ prevalence of multidrug-resistant E. coli in faecal samples of UK nursing home residents ${ }^{3}$ and a $37 \%$ prevalence of oral candida in hospitalised elderly patients. ${ }^{26}$ A meta-analysis of six trials of probiotics in critically ill patients reported that probiotics reduced colonisation with multidrug-resistant gram negatives (OR $=0.39,95 \%$ CI 0.16 to 0.95$).{ }^{27}$ Despite high prevalence of AMR colonisation in care home residents, few studies have measured the effect of probiotics on this outcome. Hatakka et al found that probiotics reduced the risk of oral candida in 276 older people by $75 \%$ $(\mathrm{OR}=0.25,95 \% \mathrm{CI} 0.10$ to 0.65$) .{ }^{28} \mathrm{Stool}$ and saliva samples at 6 to 12 months will provide $90 \%$ power at the $5 \%$ level 
to detect a $19 \%$ absolute reduction of AMR bacteria and oral candida, assuming a $30 \%$ drop-out rate.

\section{Study procedures}

The study scheme is presented in figure 1 .

\section{Data collection}

Data collection is undertaken exclusively by RNs (all fully registered with the UK Nursing and Midwifery Council) who are employed by either Cardiff University or the University of Oxford, or by local NHS Research Organisations. Care home staff were not involved in data collection.

\section{Data management}

All data collection will be by electronic data capture using a bespoke Structured Query Language (SQL) database developed by the CTR, and paper copies of all case report forms (CRFs) will be available if needed.

\section{Questionnaires}

EQ-5D (health utility) and ICECAP-O (well-being) questionnaires will be collected at baseline, 3 months and at up to 12 months.

\section{Diary}

Weekly diaries will be collated by the RNs for each participant which will include: level of dose taken of study product and method of ingestion and on what days; and if, during the past week, there have been any signs of infection; use of antibiotics; any diarrhoea; hospitalisation; any serious or trial-related adverse event. If the answer to any of the prompt questions is 'yes', further information is collected as to which days these covered, and if antibiotics were taken, the route of antibiotic administration is also recorded (eg, IV, PO, topical). Information received about antibiotic use while in hospital is captured by the RNs.

\section{Mechanistic study samples}

Full blood count and vitamin $\mathrm{D}$ will be measured at baseline in all participants who provide consent or whose consultee provides agreement for the blood test (vitamin D enhances anti-infective activities of macrophages). In a subgroup of up to 150 trial participants, additional samples will be taken at baseline and at up to 12 months to evaluate immune cell phenotypes, plasma cytokine and chemokine concentrations, cytokine and chemokine responses in whole blood samples stimulated ex vivo by toll-like receptor 2 and 4 agonists, and leucocyte phagocytosis of E. coli. A subset of patients who have been on the study product for at least 2 months will be asked to provide a blood sample on the day of (or up to 10 days prior to) and approximately 4 weeks after they receive their routine seasonal influenza vaccination. We will evaluate influenza vaccine efficacy by haemagglutination inhibition assay and antibody titres. Each participant will be asked to provide stool and saliva samples at baseline, 3 months and up to 12 months to evaluate the gut and oral microbiology. Results will be compared between those on the probiotic and placebo at the end of the study. If participants prefer not to provide any, or all, of the trial samples detailed above, this will not prevent their entry to the trial.

\section{Confidentiality}

All data will be stored confidentially on password-protected servers maintained on the Cardiff University Network.

\section{Statistical analysis}

There will not be any interim analyses. The analysis and reporting of the results will follow the Consolidated Standards of Reporting Trial guidelines. ${ }^{29}$

\section{Primary and secondary outcomes analysis}

The primary analysis will be based on a modified intention-to-treat population, with participants and their observed data included in the arm to which they were originally randomised (regardless of any postrandomisation deviations). The differences in the mean CAAD will be compared between arms using a two-level Poisson regression model, with participants nested within care homes. The CAAD count will be included as the outcome, participant gender will be included as a fixed effect (as it was an additional balancing variable at randomisation) and the length of time that participants were observed will be included as an offset variable. Negative binomial regression will be used in the presence of overdispersed count data.

The primary analysis will be by intention-to-treat and will consist of a between-group comparison of mean CAAD using Poisson regression. As the randomisation will be stratified by care home, the regression model will control for the care home a participant was recruited from. Negative binomial regression will be used in the presence of overdispersed count data. For secondary outcomes, depending on the type of data, a mixture of two-level Poisson, linear, logistic and Cox models will be used to appropriately compare trial arms with respect to rates, means, proportions and time to events.

\section{Adherence-adjusted analysis}

RNs will record day-to-day study product use on a weekly case report form. Thus, adherence data will be ascertained primarily from carer reports. We will perform adherence-adjusted analyses, deriving estimates of treatment efficacy that maintain a comparison of groups as randomised, using structural mean models. Depending on the type of outcome, these models will be fitted using the generalised methods of moments framework (for generalised linear models) or two-stage least squares procedure (for linear models). ${ }^{30}$ Two definitions of adherence will be investigated-these are based on the ABC taxonomy described by Vrijens et al. ${ }^{31}$

1. Initiation: A participant will have been deemed as having initiated their study product if the data collected on the weekly records indicates they used it at least once during the follow-up period. 


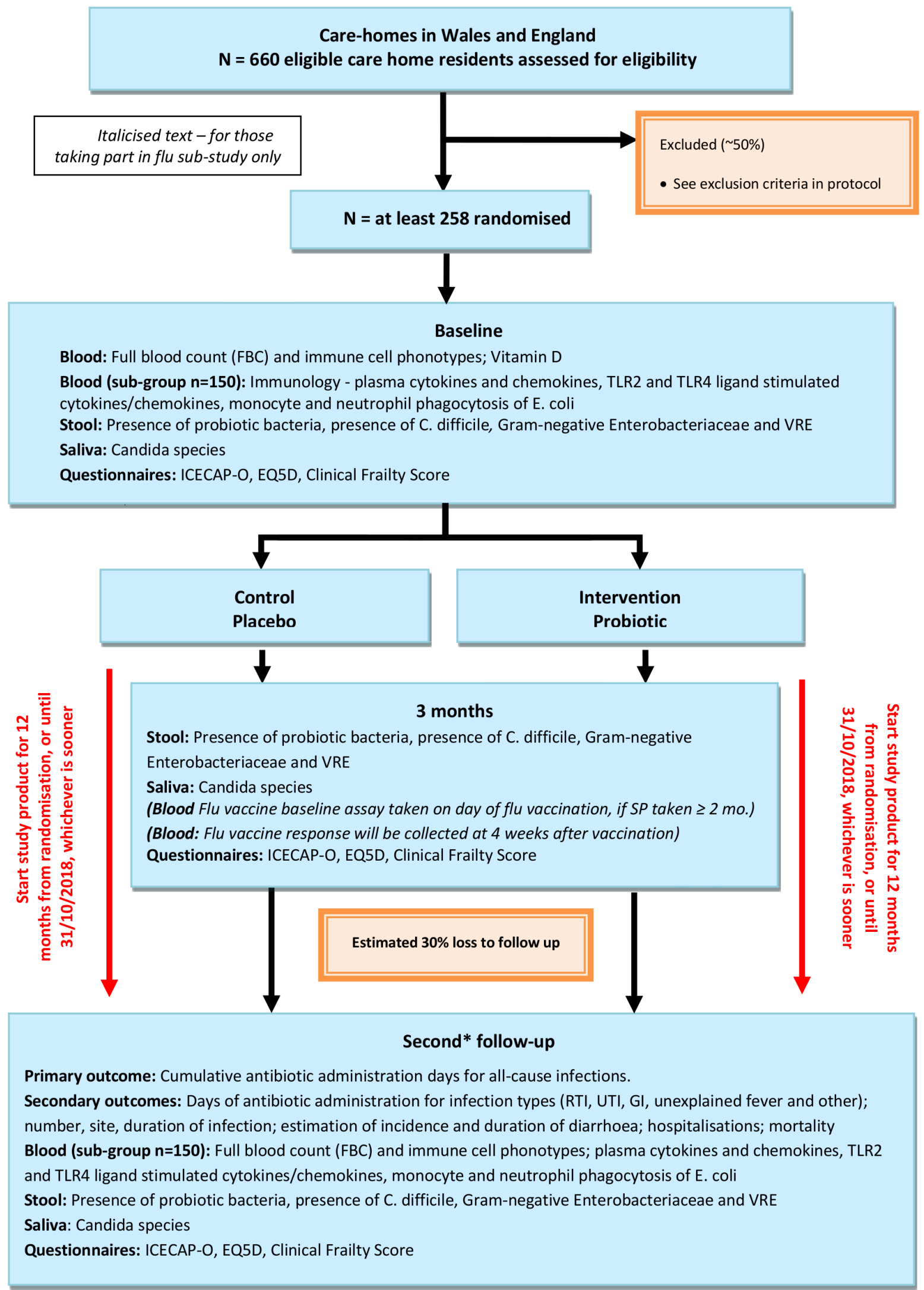

Figure 1 Scheme of study procedures and data collection for the PRINCESS trial. ${ }^{*}$ The follow-up schedule will depend on the length of time that a participant has been in the study. Where possible, participants will have a baseline assessment and follow-up at 3 and 12 months. Due to time limitations, some participants may have a truncated follow-up and will receive either a baseline assessment and 3-month follow-up or a baseline assessment, 3-month follow-up and a second follow-up between 6 and 10 months postrandomisation. Infection, antibiotic use, adverse events and study product use will be recorded at regular intervals by the RN from care home notes for up to 12 months postrandomisation or until 31 October 2018, whichever is sooner. 
2. Implementation: Percentage of days that participants took their study product as indicated (with those not initiating set at zero).

\section{Missing data adjustments}

Given our proposed intensive ascertainment processes, missing data are likely to be minimal for participants who remain in the trial for the full duration. Where missing data are likely to occur, it will most likely be due to participant dropout, with reasons for dropout falling into two broad categories, withdrawal from the trial and death. We will investigate the sensitivity of our findings to various missing data mechanisms, exploring the extent to which withdrawal (and within this health-related withdrawal and withdrawal for other reasons) and death (and within this infection-related death and death due to other causes) require different adjustment methods.

\section{Subgroup analysis}

Subgroup analyses will be performed to explore differential treatment effects on the primary outcome measure. Interaction terms will be fitted between trial arm and the following measures thought to be correlated with the primary outcomes. These include the following:

- Gender.

- Baseline capacity to consent to the trial.

- Clinical frailty scale at baseline (grouped as very fit to managing well/vulnerable to moderately frail/ severely frail to terminally ill).

\section{Mechanism analysis}

Data from the mechanistic studies will be compared between groups controlling for value at study entry. Further statistical modelling will explore the causal mechanisms by which the probiotic may have an effect. Mediation analyses will explore the effect of exposure to probiotics on CAAD and cumulative number of infection days and whether this is mediated through an effect on antimicrobial resistance. These analyses will be performed using G-computation. ${ }^{32} 33$

A detailed Statistical Analysis Plan (SAP) will be written and signed prior to any analysis commencing.

\section{Trial Steering Committee and Independent Data Monitoring Committee}

An independent Trial Steering Committee (TSC) will be established to provide oversight of the PRINCESS trial. This will include at least an independent chairperson, two independent members and a patient representative. An Independent Data Monitoring Committee (IDMC) will convene at least annually to provide oversight of all matters relating to patient safety and data quality. Members will be required to sign up to the remit and conditions as set out in the TSC and IDMC Charters.

\section{Adverse event reporting \\ Non-serious adverse events (AEs)}

Non-serious AEs with the causality classification of probably or definitely related to the study product (such as gastrointestinal symptoms or ingredient-related allergic reaction) or study procedures (such as a haematoma at the site of venepuncture for a study sample) will be collected as part of routine follow-up (recorded by the $\mathrm{RN}$ as part of the weekly review) from the time of consent until the end of the trial. Other non-serious AEs will not be collected. The principal investigator/care home staff will manage AEs according to routine care home procedures.

\section{Serious adverse events}

Serious adverse events (SAEs) will be collected as part of routine follow-up (from the time of consent until the end of the trial). SAEs will be discussed (in person, by phone, or by email) by the RN with a second delegated assessor (eg, another $\mathrm{RN}$ or a clinician involved in the PRINCESS study) to confirm the causality classification (definitely, probably, possibly, unlikely, unrelated). The details of the second assessment will be recorded as part of the weekly review. Where there is a difference in classification between the two assessors, the highest category of causality (most likely to be related) will be selected. If either reviewer classifies the SAE as either definitely or probably related to study procedure or study intervention, a Serious Adverse Reaction (SAR) form will be completed and the SAR will be assessed for expectedness by the chief investigator or delegated clinical reviewer.

- If the event is classified as definitely or probably related to the study procedure and is unexpected, it will be reported to sponsor, Research Ethics Committee (REC) and the IDMC.

- If the event is classified as definitely or probably related to the study intervention, is unexpected and unblinding shows that the participant is allocated to the intervention arm, it will be reported to the sponsor, REC and the IDMC.

- If the event is classified as definitely or probably related to the study intervention, is unexpected and unblinding shows that the participant is allocated to the placebo arm, and the clinical reviewer believes the SAR to be an allergic reaction to the excipient, it will be reported to the sponsor, REC and the IDMC.

\section{ETHICS AND DISSEMINATION \\ Ethical approval}

All trial participants or their consultee will give informed consent or consultee declaration before taking part (see earlier).

The following substantial amendments were made to the trial and were communicated to all trial sites:

1. Changes to the protocol.

2. Trial poster for care homes.

3. Addition of a secondary outcome (incidence and duration of diarrhoea).

4. Letter to consultee of a trial participant who initially had capacity, but who lost capacity.

5. Consent procedure for nominated consultee.

6. Shortened follow-up and clarification of objective and outcome terminologies. 


\section{Dissemination}

The final report will be prepared for the National Institute of Health Research (NIHR) Journal series. The main trial results will be submitted for publication in a first rank, high-impact, international, peer-reviewed general medical journal. Other results, including secondary and mechanistic analyses, will also be submitted to high-impact, peer-reviewed scientific journals. Results will also be presented at national and international meetings, including general medical, geriatric medicine and primary care meetings. We will also disseminate our results to the Department of Health, NHS bodies in the four UK countries, NICE, NHS Evidence, Age UK and other patient and policy stakeholders. Dissemination of our findings will have two main objectives:

1 . To use any recognised benefits of the interventions resulting from this study and further evaluation.

2. To enhance academic collaborations linking relevant research areas in this field and identify further areas where research could be effectively directed.

We will hold panel discussion events inviting academics, stakeholders, members of the public and service users at the end of the project to inform further course of action, which will depend on our findings. Our Involving People contributors will lead on developing a dissemination strategy most appropriate for care home staff, care home residents and their families.

\section{DISCUSSION}

Interventions that reduce antibiotic use, incidence and severity of infections and antimicrobial resistance in care home residents have the potential to improve health, save costs and help preserve the efficacy of existing antibiotics. Currently, infections are the the most common reason for the hospitalisation of care home residents, and at a time when we are experiencing AMR because of antibiotic overuse, there is urgent need to investigate safe and effective alternatives for preventing infections and enhancing immunity. Probiotics are cheap, safe and have minimal side effects, and there are plausible mechanisms by which they may enhance resilience against infection in care home residents and thus reduce antibiotic use. This could lead in turn to containing antibiotic resistance and reduce the risk of side effects from antibiotics. However, empirical evidence is needed to determine whether care home residents should take probiotics to reduce antibiotic use and prevent infection. This study will also add to the evidence base about the influence of taking probiotic on measures of immunity, including response to seasonal influenza vaccine. The PRINCESS trial will determine the efficacy of probiotics to reduce antibiotic use and infections in care home residents with potential benefits to the community and healthcare delivery. A lay summary of the results and links to publications will be made available on the University trial website and will be given to the care home, the participant and/or their consultee.

\section{Author affiliations}

${ }^{1}$ Centre for Trials Research, Cardiff University, Cardiff, UK

${ }^{2}$ Primary Care and Population Sciences, University of Southampton, Aldermoor Health Centre, Southampton, UK

${ }^{3}$ Division of Population Medicine, School of Medicine, Neuadd Meirionnydd, Cardiff University, Cardiff, UK

${ }^{4}$ Human Development \& Health, Faculty of Medicine, University of Southampton, Southampton, UK

${ }^{5} \mathrm{NIHR}$ Southampton Biomedical Research Centre, University of Southampton NHS Foundation Trust and University of Southampton, Southampton, UK

${ }^{6}$ Clinical Trials Unit, Nuffield Department of Primary Care Health Sciences, University of Oxford, Oxford, UK

${ }^{7}$ Lay Representative, Cardiff, UK

${ }^{8}$ Specialist Antimicrobial Chemotherapy Unit, Public Health Wales Microbiology Cardiff, University Hospital of Wales, Cardiff, UK

Acknowledgements Professor George Lewith (deceased 17 March 2017) helped in the development of the research question, study design, obtaining the funding and implementation of the study protocol. Catherine Lisles is responsible for data management. The authors would like to thank Ms Jennifer Richards and Ms Leanne Davies who are responsible for processing the microbiology samples, and Dr Elizabeth A Miles and Ms Vivian Castro Herrera for conducting the immune mechanism research. The Centre for Trials Research receives funding from Health and Care Research Wales and Cancer Research UK. FDRH acknowledges his partfunding from the National Institute for Health Research (NIHR) School for Primary Care Research, the NIHR Collaboration for Leadership in Health Research and Care (CLARHC) Oxford, the NIHR Oxford Biomedical Research Centre (BRC,) UHT, and the NIHR Oxford Medtech and In-Vitro Diagnostics Cooperative (MIC). The authors would also like to acknowledge the contribution of the Trial Steering Committee members, namely Professor Steve lliffe, Dr Glenn R Gibson, Dr Jonathan Sandoe, Dr Meena Rafiq, Robin Willmott and the members of the Independent Data Monitoring Committee, namely Dr Stephen Bremner, Dr Neil Haslam and Dr Matthew Ridd.

Contributors CCB is the Chief Investigator of this trial. CCB led the development of the research question, study design, obtaining the funding and implementation of the study protocol, along with TB, PCC, JDo, NAF, RF, DG, RH, KH, PL, ML, MM, VS, AT and MW. EO- $J$ is the Trial Manager and RL the Senior Trial Manager who coordinated the operational delivery of the study protocol and recruitment. JD and AE provide research nurse support. DG and MLau are the trial statisticians. MD is the Trial Manager. KA and HS are the data managers. All authors listed provided critical review and final approval of the manuscript.

Funding This work was supported by the Efficacy and Mechanism Evaluation programme, which is funded by the MRC and NIHR, with contributions from CSO in Scotland, HCRW in Wales and the HSC R\&D, Public Health Agency in Northern Ireland. This project is managed by the NIHR Evaluation, Trials and Studies Coordination Centre (NETSCC) (Efficacy and Mechanism Evaluation (EME), grant number 13/95/10 - Probiotics to Reduce Infections iN CarE home reSidentS (PRINCESS)). The views expressed in this publication are those of the author(s) and not necessarily those of the MRC, NHS, the National Institute for Health Research or the Department of Health. Cardiff University, Research and Innovation Services Department, Contracts Team, Cardiff University, 30-36 Newport Road, Cardiff, CF24 ODE (SPON1426-15).

Competing interests PCC reports grants from National Institute for Health Research, grants from EME Programme funded by NIHR / MRC. During the conduct of the study PCC receives personal fees from Christian Hansen, and non-financial support from Christian Hansen outside the submitted work.

Patient consent for publication Not required.

Ethics approval The Wales REC 3 has approved the study (15/WA/0306) on 13 November 2015.

Provenance and peer review Not commissioned; externally peer reviewed.

Open access This is an open access article distributed in accordance with the Creative Commons Attribution Non Commercial (CC BY-NC 4.0) license, which permits others to distribute, remix, adapt, build upon this work non-commercially, and license their derivative works on different terms, provided the original work is properly cited, appropriate credit is given, any changes made indicated, and the use is non-commercial. See: http:// creativecommons.org/licenses/by-nc/4.0/. 


\section{REFERENCES}

1. Bonomo RA. Multiple antibiotic-resistant bacteria in long-term-care facilities: An emerging problem in the practice of infectious diseases. Clin Infect Dis 2000;31:1414-22.

2. Hood K, Nuttall J, Gillespie D, et al. Probiotics for AntibioticAssociated Diarrhoea (PAAD): a prospective observational study of antibiotic-associated diarrhoea (including Clostridium difficileassociated diarrhoea) in care homes. Health Technol Assess 2014;18:1-84.

3. Rooney PJ, O'Leary MC, Loughrey AC, et al. Nursing homes as a reservoir of extended-spectrum beta-lactamase (ESBL)-producing ciprofloxacin-resistant Escherichia coli. J Antimicrob Chemother 2009;64:635-41.

4. Heginbothom MCM, Howe R, Davies E. Antibacterial Usage in Primary Care In Wales 2013/14 - 2017/18 Public Health Wales, 2018 01/08/2018. Report No.: Final v1. 2018

5. Vihta KD, Stoesser N, Llewelyn MJ, et al. Trends over time in Escherichia coli bloodstream infections, urinary tract infections, and antibiotic susceptibilities in Oxfordshire, UK, 1998-2016: a study of electronic health records. Lancet Infect Dis 2018;18:1138-49.

6. Costelloe C, Metcalfe C, Lovering A, et al. Effect of antibiotic prescribing in primary care on antimicrobial resistance in individual patients: systematic review and meta-analysis. BMJ 2010;340:c2096.

7. van Hecke O, Wang $\mathrm{K}$, Lee JJ, et al. Implications of Antibiotic Resistance for Patients' Recovery From Common Infections in the Community: A Systematic Review and Meta-analysis. Clin Infect Dis 2017;65:371-82.

8. Moro-García MA, Alonso-Arias R, Baltadjieva M, et al. Oral supplementation with Lactobacillus delbrueckii subsp. bulgaricus 8481 enhances systemic immunity in elderly subjects. Age 2013;35:1311-26.

9. O'Neill J. Antimicrobial Resistance: Tackling a crisis for the health and wealth of nations. 2014.

10. Health ECoEo, Bacteria NPoPiFiPMwLLA. Health and Nutritional Properties of Probiotics in Food Including Powder Milk with Live Lactic Acid Bacteria: Report of a Joint FAO WHO Expert Consultation on Evaluation of Health and Nutritional Properties of Probiotics in Food Including Powder Milk with Live Lactic Acid Bacteria, American Córdoba Park Hotel, Córdoba, Argentina, 1 - 4 October 2001. 2001

11. Boge T, Rémigy $M$, Vaudaine $S$, et al. A probiotic fermented dairy drink improves antibody response to influenza vaccination in the elderly in two randomised controlled trials. Vaccine 2009;27:5677-84.

12. Lomax A, Calder P, Probiotics CPC. immune function, infection and inflammation: a review of the evidence from studies conducted in humans. Curr Pharm Des 2009;15:1428-518.

13. Maidens $C$, Childs $C$, Przemska $A$, et al. Modulation of vaccine response by concomitant probiotic administration. Br J Clin Pharmacol 2013;75:663-70.

14. Zaky A. Interventions to prevent the acquisition of resistant Gramnegative bacteria in critically ill patients- $A$ systematic review and meta-analysis [Master's]. University of Washington, 2012.

15. Hempel S, Newberry SJ, Maher AR, et al. Probiotics for the prevention and treatment of antibiotic-associated diarrhea: a systematic review and meta-analysis. Jama 2012;307:1959-69.
16. Snydman DR. The safety of probiotics. Clin Infect Dis 2008;46(s2):S1 04-S111. discussion S44-51.

17. Davidson LE, Fiorino A-M, Snydman DR, et al. Lactobacillus GG as an immune adjuvant for live-attenuated influenza vaccine in healthy adults: a randomized double-blind placebo-controlled trial. Eur J Clin Nutr 2011;65:501-7.

18. Toiviainen A, Jalasvuori $\mathrm{H}$, Lahti $\mathrm{E}$, et al. Impact of orally administered lozenges with Lactobacillus rhamnosus $\mathrm{GG}$ and Bifidobacterium animalis subsp. lactis BB-12 on the number of salivary mutans streptococci, amount of plaque, gingival inflammation and the oral microbiome in healthy adults. Clin Oral Investig 2015;19:77-83.

19. Liu PT, Krutzik SR, Modlin RL. Therapeutic implications of the TLR and VDR partnership. Trends Mol Med 2007;13:117-24.

20. Np L, Litonjua A, Gold DR, et al. Gut microbiota, probiotics, and vitamin D: interrelated exposures influencing allergy, asthma, and obesity? J Allergy Clin Immunol 2011;127:1087-94. quiz 95-6.

21. Smith TJ, Rigassio-Radler D, Denmark R, et al. Effect of Lactobacillus rhamnosus LGG ${ }^{\circledR}$ and Bifidobacterium animalis ssp. lactis BB-12 ${ }^{\circledR}$ on health-related quality of life in college students affected by upper respiratory infections. Br J Nutr 2013;109:1999-2007.

22. Taipale $\mathrm{T}$, Pienihäkkinen $\mathrm{K}$, Isolauri $\mathrm{E}$, et al. Bifidobacterium animalis subsp. lactis BB-12 in reducing the risk of infections in infancy. $\mathrm{Br} J$ Nutr 2011;105:409-16.

23. Rautava S, Salminen S, Isolauri E. Specific probiotics in reducing the risk of acute infections in infancy - a randomised, double-blind, placebo-controlled study. Br J Nutr 2009;101:1722-6.

24. Kajander K, Myllyluoma E, Rajilic-Stojanovic M, et al. Clinical trial: multispecies probiotic supplementation alleviates the symptoms of irritable bowel syndrome and stabilizes intestinal microbiota. Aliment Pharmacol Ther 2008;27:48-57.

25. Butler CC, Duncan D, Hood K. Does taking probiotics routinely with antibiotics prevent antibiotic associated diarrhoea? BMJ 2012;344:e682

26. Lingard L. Health Needs Assessment of the Residents of Nursing Homes in South Tyneside Executive Summary 2011. 2011. Report No.

27. McFarland LV. Evidence-based review of probiotics for antibioticassociated diarrhea and Clostridium difficile infections. Anaerobe 2009;15:274-80.

28. Hatakka K, Ahola AJ, Yli-Knuuttila H, et al. Probiotics reduce the prevalence of oral candida in the elderly--a randomized controlled trial. J Dent Res 2007;86:125-30.

29. Schulz KF, Altman DG, Moher D. CONSORT 2010 Statement: updated guidelines for reporting parallel group randomised trials. BMC Med 2010;8:18.

30. Vansteelandt S, Goetghebeur E. Causal inference with generalized structural mean models. J Royal Stat Soc 2003;65:817-35.

31. Vrijens B, De Geest S, Hughes DA, et al. A new taxonomy for describing and defining adherence to medications. $\mathrm{Br} J$ Clin Pharmacol 2012;73:691-705.

32. Robins JM HM. In: Fitzmaurice GDM, Verbeke G, Molenberghs G, eds. Estimation of the causal effects of time-varying exposures. New York: Chapman and Hall/CRC Press, 2009.

33. Daniel RM, De Stavola BL, Cousens SN. Gformula: Estimating Causal Effects in the Presence of Time-Varying Confounding or Mediation using the G-Computation Formula. Stata J 2011;11:479-517. 